of each organization, and their relation to each other, by creating a matrix of the functions and organizations.

Background: When a natural disaster or mass-casualty incident occurs, a large number of injured people visit hospitals. During these times, hospitals need to provide additional services. Thus, it is necessary for various related organizations such as hospitals, municipalities, medical associations, and trade associations, to collaborate. There is a pressing need to establish an Area Disaster Resilience Management System for Healthcare (ADRMS-H) to increase medical resilience. It is necessary to identify the functions of, and coordination between, organizations needed to develop ADRMS-H.

Methods: We identified the medical care requirements during a disaster and the functions that guarantee ongoing health care by investigating disaster records and interviewing several doctors who provided health care services during the Great East Japan Earthquake. In addition, we analyzed the changes in the functions by the hour, and divided the phases based on the changes. Furthermore, we identified the organizations that are needed to fulfill each function and created a matrix between the functions and organizations of each phase.

Results: We created the matrix based on each of the seven phases. The functions included those that must be fulfilled by hospitals, such as providing treatment, and those that must be fulfilled by municipalities, and so on, such as establishing aid stations. Some functions are fulfilled in cooperation with various organizations.

Conclusion: When large earthquakes occurred in Japan, it was difficult to understand the functions needed to ensure continued health care services. Creating the matrix of each phase enables us to understand the changing roles of each organization by the hour. This facilitates in the establishment of ADRMS-H.

Prehosp Disaster Med 2017;32(Suppl. 1):s54-s55

doi:10.1017/S1049023X17001510

\section{A Systematic Review of Human Health following Flood and} Storm Disasters

Dell Saulnier, Kim Brolin Ribacke, Johan Von Schreeb

Department Of Public Health Sciences, Karolinska Institutet, Stockholm/Sweden

Study/Objective: The objective of this review was to elucidate the health problems over time, following flood and storm disasters.

Background: The health care response to a flood or storm disaster should be guided by the expected health needs of the affected population, for both existing conditions and those caused by the disaster. It is essential to know how the burden of disease varies during different phases after the disaster, but there are few studies on the quantifiable changes in health, or in comparing the difference between floods and storms.

Methods: A literature search of the databases Medline, Cinahl, Global Health, Web of Science Core Collection, Embase, and PubMed was conducted in June 2015, for English-language research articles on morbidity or mortality and flood or storm disasters. Articles on mental health, interventions, and health care workers were excluded. Data were extracted from articles that met the eligibility criteria and analyzed by narrative synthesis.

Results: The review included 113 studies. Poisonings, wounds, gastrointestinal infections, and skin or soft tissue infections all increased after storms. Gastrointestinal infections were more frequent after floods. Leptospirosis and diabetes-related complications increased after both. The majority of changes occurred within four weeks of floods or storms.

Conclusion: Health changes differently after floods and after storms. There is a lack of data on the health effects of floods alone, long-term changes in health, and the strength of the association between the disaster and health problems. An analysis of how contextual factors affect health problems would be a useful complement to the results. The review highlights areas of consideration for medical response, and the need for high quality, systematic research in this area. The study was funded by the Swedish National Board of Health and Welfare.

Prehosp Disaster Med 2017;32(Suppl. 1):s55

doi:10.1017/S1049023X17001522

\section{Mass Fatality Management in the US}

Robyn R. Gershon ${ }^{1}, Q_{i} Z^{2} i^{2}$, Ezinne Nwankwo ${ }^{2}$, Jacquenline A. Merrill ${ }^{3}$

1. Institute For Health Policy Studies, Sch Of Medicine, University of California, San Francisco, San Francisco/United States of America

2. Institute For Health Policy Studies, Sch Of Medicine, University of California, San Francisco, San Francisco/CA/United States of America

3. School Of Nursing And Department Of Biomedical Informatics, Columbia University, New York/NY/United States of America

Study/Objective: This study was conducted to assess the infrastructure's capacity and capability to effectively respond to Mass Fatality Incidents (MFI).

Background: In recognition of the increasing incidence of Mass Fatality Incidents (MFI), a large and complex multi-level infrastructure has been developed in the US to prepare and respond to these types of disaster events.

Methods: In 2013, anonymous online surveys were distributed to representatives of 5 key sectors comprising the MFI infrastructure (medical examiners/coroners, the death care industry, health departments, faith-based organizations, and offices of emergency management). Three new metrics were developed to measure "preparedness:" (1) organizational capacity, (2) operational capability, and (3) resource sharing capability (between response partners).

Results: A total of 879 respondents reported highly variable organizational capacity: $15 \%$ had previously responded to mass fatality incidents (MFI); $42 \%$ reported staff trained for MFI, but only $27 \%$ for complex MFI (ie. involving hazardous contaminants). Less than half (48\%) participated in jurisdictionwide MFI drills. An estimated $75 \%$ of staff would be willing and able to report to duty for MFI, but that declined to $53 \%$ if contaminants were involved. Most perceived their organization as "somewhat prepared," but 13\% indicated "not at all." Fewer than 25 additional fatalities in a 48 -hr period would exceed 
existing capacity to respond. Operational capability scores ranged from 33\% (death care industry) to $77 \%$ (offices of emergency management). Resource sharing capability analysis indicated that only $42 \%$ of possible reciprocal relationships between resource-sharing partners were present. The overall cross-sector composite score was $51 \%$; that is, half of the key capabilities for preparedness were in place.

Conclusion: Results indicate that the US mass fatality infrastructure is sub-optimally prepared for MFI that exceeds 25 or fewer additional deaths in a 48 - $\mathrm{hr}$ period. National leadership is needed to ensure sector-specific and infrastructure-wide preparedness, with a special focus on training, drills, and planning activities for large-scale or complex MFI.

Prehosp Disaster Med 2017;32(Suppl. 1):555-s56

doi:10.1017/S1049023X17001534

Preparedness of US Health Care Volunteers Who Deployed to the West Africa Ebola Epidemic

Robyn R. Gershon ${ }^{1}$, Liza A. Dernehl' ${ }^{2}$ Ezinne Nwankwo ${ }^{3}$, Qi Zhi ${ }^{3}$, Kristine A. Qureshi ${ }^{4}$

1. Institute For Health Policy Studies, Sch Of Medicine, University of California, San Francisco, San Francisco/United States of America

2. Graduate School Division, University of California, San Francisco, San Francisco/CA/United States of America

3. Institute For Health Policy Studies, Sch Of Medicine, University of California, San Francisco, San Francisco/CA/United States of America

4. School Of Nursing, University of Hawaii at Manoa, Honolulu/HI/ United States of America

Study/Objective: To identify the preparedness of US health care volunteers for hot zone (West Africa Ebola) deployment.

Background: Each year, an estimated 200,000 US health care workers voluntarily deploy to provide care and expertise to disaster events worldwide. Many of these involve bioevents (outbreaks, epidemics, and pandemics), and sometimes these bioevents involve extremely dangerous and novel pathogens. The preparedness of these volunteers to work in high risk "hot zones," had not, to our knowledge, been previously assessed.

Methods: In 2015, a sample of 16 US health care volunteers who had recently returned from West Africa were recruited for qualitative interviews. Data on preparedness for each phase of deployment (pre, peri, and post) was collected and analyzed using thematic analysis and constant comparison methodology. Results: Prior to deployment, most participants reported very limited preparation for the deployment. Training, especially in the early days of the epidemic, was highly variable, and in some cases consisted of simply reading a manual on lethal viruses. During the deployment, extreme resource limitations and poor management of the mission was a serious source of frustration and concern. The necessity for altered standards of care delivery was also very troubling. Upon return home, participants were unprepared for the negative reactions and resentment of their friends and family members. The isolation they felt during the quarantine period was reported as one of the most stressful aspects of the entire experience. Depression, stigmatization, and interpersonal difficulties were also common upon return to the US.
Conclusion: Preparedness of healthcare volunteers was suboptimal at each stage of deployment. All stakeholders, including volunteers, sponsoring organizations, government agencies, and professional organizations have a shared responsibility in ensuring that volunteers to medical missions are adequately prepared.

Prehosp Disaster Med 2017;32(Suppl. 1):s56

doi:10.1017/S1049023X17001546

\section{Health Emergency Operation Center to Face Public Health Events in Africa: Senegalese Experience \\ Abdoulaye Bousso \\ Health Emergency Operation Center, Ministry of Health, Senegal, Dakar/Senegal}

Study/Objective: To share the experience of a low-income country on setting up a Heath Emergency Operation Center (HEOC) to face health threats.

Background: The last Ebola outbreak in West Africa was a great alert for our countries on the importance of preparedness, and to face public health events with international concern. For Senegal, after managing our imported case, our big lesson learned was to establish a national structure, which can involve an all-emergency management cycle. It's why we set up a HEOC; the HEOC is in charge of all health events, beyond epidemics.

Methods: The HEOC was established in December 2014. A participative approach was developed during the process of setting up, with the ministry of health, other ministries and partners, which was part of the process.

Results: The HEOC brought some added value:

- Coordination: the incident management system is now adopted for the management of emergencies and disasters.

- Plans and procedures have been developed, for the HEOC and for some risk

- Exercises and drills were conducted to test SOPs and the response efficiency

- One health approach was adopted.

Conclusion: Shared experiences of a low-income country, on setting up a Health Emergency Operation Center (HEOC) to face health threats.

Prehosp Disaster Med 2017;32(Suppl. 1):s56

doi:10.1017/S1049023X17001558

\section{Hazard Vulnerability Analysis: Practices in Boston}

Hospitals

Douglas A. Romney ${ }^{1}$, Meg S. Femino ${ }^{2}$, Ritu R. Sarin ${ }^{1}$, Michael S. Molloy, Amalia Voskanyan ${ }^{1}$, Gregory R. Ciottone ${ }^{1}$

1. Department Of Emergency Medicine, BIDMC Fellowship in Disaster Medicine, Boston/MA/United States of America

2. Emergency Management, Beth Israel Deaconess Medical Center, Boston/MA/United States of America

Study/Objective: To determine what instruments and methods Boston hospitals and hospital systems use to perform Hazard Vulnerability Analysis (HVA). 